\title{
Magnetite epitaxial growth on Ag(001): selected orientation, seed layer, and interface sharpness.
}

\author{
A. D. Lamirand, ${ }^{\mathrm{a}, \mathrm{b}}$ S. Grenier, ${ }^{\mathrm{a}, \mathrm{b}}$ V. Langlais, ${ }^{\mathrm{c}}$ A. Y. Ramos, ${ }^{\mathrm{a}, \mathrm{b}}$ H. C. N. Tolentino, ${ }^{\mathrm{a}, \mathrm{b}}$ X. \\ Torrelles, ${ }^{\mathrm{d}}$ M. De Santis ${ }^{\mathrm{a}, \mathrm{b},{ }^{*}}$ \\ aUniversité Grenoble Alpes, Institut Néel, F-38042 Grenoble, France \\ ${ }^{\mathrm{b} C N R S}$, Institut Néel, F-38042 Grenoble, France \\ 'CNRS, CEMES (Centre d'Elaboration des Matériaux et d'Etudes Structurales), B.P. 94347, 29 \\ rue Jeanne Marvig, F-31055 Toulouse, France \\ ${ }^{\mathrm{d}}$ Institut de Ciència de Materials de Barcelona, ICMAB-CSIC, Bellaterra, 08193 Barcelona, \\ Spain
}

\begin{abstract}
Epitaxial iron oxide layers with different orientations were grown on $\mathrm{Ag}(001)$ surface by choosing the appropriate preparation conditions. A film with a hexagonal surface mesh interpreted as (111)oriented magnetite was formed by reactive deposition of iron in molecular oxygen at room temperature (RT), followed by annealing in UHV. Instead highly ordered epitaxial layers with P4m symmetry were obtained by a three steps process, optimized through in situ experiments. Following this method, an ultrathin Fe layer was first grown in coherent epitaxy on the substrate, and then dosed twice with $\mathrm{O}_{2}$, first at RT and next during annealing. A structural analysis combining lowenergy electron diffraction, scanning tunneling microscopy and accurate surface $\mathrm{x}$-ray diffraction measurements confirmed that these films consist of (001)-oriented magnetite, although with a slight tetragonal distortion induced by the substrate constraints. Both its surface and interface are atomically sharp, an essential requirement for its integration into spintronic based devices.
\end{abstract}

Keywords: Magnetite films; Epitaxial growth; Surface x-ray diffraction; ultrathin transition metal oxide films.

\section{Introduction}

Transition metal oxides (TMO) films are extensively studied in view of their integration in heterostructures. Of particular interest are magnetic TMO that can play the role of active elements in spin dependent electronic devices. The density of state (DOS) in such TMO can either exhibit a spin dependent gap or be spin polarized at the Fermi level. Thin films of these oxides can be then used for spin filtering $[1,2]$ or spin injection, respectively. The properties of TMO multilayers critically depend on the electronic and magnetic interactions at the interfaces, and hence on their detailed atomic structure.

$\mathrm{Fe}_{3} \mathrm{O}_{4}$ (magnetite) is a prominent material for spintronic applications. It is one of the three ironoxide phases observed in nature together with $\mathrm{FeO}$ (wüstite) and $\alpha-\mathrm{Fe}_{2} \mathrm{O}_{3}$ (hematite). Two further phases, $\gamma-\mathrm{Fe}_{2} \mathrm{O}_{3}$ (maghemite) [3] and $\varepsilon-\mathrm{Fe}_{2} \mathrm{O}_{3}[4,5]$ have been artificially synthesized. Under 
thermodynamic equilibrium, their stability range depends on the ambient $\mathrm{O}_{2}$ gas pressure and on the temperature [6].

From an historical point of view magnetite is the first known magnetic material and has been deeply investigated on all its aspects. Above the Verwey transition [7], occurring at about $120 \mathrm{~K}$, magnetite crystallizes in the cubic inverse spinel structure with space group $\mathrm{Fd} 3 \mathrm{~m}\left(a_{\mathrm{Fe} 304}=839.6 \mathrm{pm}\right)$. The iron cations $\left(\mathrm{Fe}^{2+}\right.$ and $\mathrm{Fe}^{3+}$ ) are located in interstitial octahedral and tetrahedral sites of a closepacked face-centered cubic (fcc) sublattice which is formed by the oxygen anions [8]. Magnetite is expected to be a half-metal, i.e. with DOS of only one spin orientation crossing the Fermi level [9], and exhibits a high Curie temperature (858 K). Its use as electrode in magnetic tunnel junction (MTJ) is therefore very appealing, and a very high magnetoresistance (MR) is expected [10]. However measurements on MTJ with magnetite electrodes grown both by sputtering and molecular beam epitaxy (MBE) techniques show MR values well below the expectations [11-17]. Several explanations have been proposed for this quite disappointing finding. A non-stoichiometric region close to the interface with the presence of defects or/and different phases would result in a magnetic dead layer and/or spin flip processes at the interface. The presence and the role of antiphase domain boundaries (APBs) in the degradation of the magnetic and transport properties of ultra-thin films have been pointed out by several authors [18-21]. APBs form in $\mathrm{Fe}_{3} \mathrm{O}_{4} / \mathrm{MgO}(001)$ system due to the lower film symmetry [22]. They can result in antiferromagnetic coupling at the boundary which reduces the total spin polarization of the film [23].

In surface science fcc (001) metallic surfaces have been largely employed as substrates for growing TMO films. It was found that strain is a crucial parameter in determining the epitaxial relationships. For example CoO films grow (001) oriented on $\mathrm{Ag}(001)$ [24] while for larger strains, as in the case of CoO/Ir(100), (111) films are usually obtained [25]. For the latter system however it was shown that the CoO orientation can be tuned to (001) by controlling the chemistry at the interface [26]. The switching is achieved by the deposition of a Co buffer layer about 2 monolayers thick on the clean non-reconstructed surface. This layer is pseudomorphic, and after a moderate oxidation it forms a $\mathrm{c}(4 \mathrm{x} 2)-\mathrm{Co}_{3} \mathrm{O}_{4} / \mathrm{Co} / \mathrm{Ir}(001)$ surface, precursor for the growth of $\mathrm{CoO}(001)$. We employed a similar method for growing high quality (001) magnetite ultrathin films on $\mathrm{Ag}(001)$.

The formation of iron oxides on $\mathrm{Ag}(001)$ has already been investigated, evidencing different phases as function of the $\mathrm{O}_{2}$ pressure during annealing [27]. A possible drawback with this substrate is the presence of a moderate intermixing at the interface during Fe/Ag(001) growth at room temperature [28]. This could result in surface segregation and in a rough interface after annealing in oxygen. A critical achievement in our experiment is the growth and characterization of a single phase oxide film. Magnetite and maghemite share the same crystal symmetry and possess very close lattice constants $\left(a_{\gamma \text {-Fe2O3 }}=833 \mathrm{pm}\right)$ [29]. Maghemite structure is obtained by introducing Fe vacancies on the $\mathrm{Fe}_{3} \mathrm{O}_{4}$ spinel octahedral site. Distinguishing these two oxides by diffraction techniques is tricky, and requires accurate measurements. The optimization of the growing conditions discussed here is based on in situ experiments and aims to pure magnetite ultrathin films with sharp interfaces.

\section{Experimental set-ups}

Two distinct experimental set-ups were used for this study. Both are fully equipped for sample preparation and analysis in ultra-high vacuum (UHV) environment. The first one, located at the Néel Institute, possesses a commercial scanning tunneling microscope (Omicron VT STM/AFM), 
a low-energy electron diffractometer (LEED) and an Auger electron spectrometer (AES). The second one, located at the French CRG BM32 beamline of the European Synchrotron Radiation Facility (ESRF), is mounted on a Z-axis diffractometer (with additional degrees of freedom to align the sample normal) and is dedicated to in situ surface x-ray diffraction (SXRD) [30]. The oxide layers were grown on a $\mathrm{Ag}(001)$ single crystal with a miscut $<0.1^{\circ}$, previously cleaned by repeated cycles of $\mathrm{Ar}+$ ion sputtering followed by annealing at about $850 \mathrm{~K}$. The substrate temperature was measured using an infrared pyrometer. Cleanliness was checked by AES. Iron was evaporated from a pure rod using a water-cooled electron-beam evaporator. The base pressure was in the low $10^{-11}$ $\left(10^{-10}\right)$ mbar range for the STM (SXRD) set-up. The Fe deposition rate was typically 1 monolayer (ML) per 5 minutes, calibrated with a quartz micro-balance. STM images were obtained in constant current mode, with the voltage bias $\left(\mathrm{V}_{\mathrm{s}}\right)$ applied to the sample. The SXRD measurements were performed at photon energy of $22 \mathrm{keV}$ and with an incident angle $\alpha_{\mathrm{i}}$ of the x-ray settled at $0.48^{\circ}$, three times the substrate critical angle for total reflection. The reciprocal space unit vectors of the Ag (001) surface unit cell $\left(\overrightarrow{a_{S}^{1}}=\frac{a_{A g}}{2}[1 \overline{10}], \overrightarrow{a_{S}^{2}}=\frac{a_{A g}}{2}[110]\right.$, and $\left.\overrightarrow{a_{s}^{3}}=a_{A g}[001]\right)$ will be used to index both the in-plane position of LEED spots and the momentum transfer in SXRD.

In the following we will discuss the results obtained by two different preparation methods. Sample A was grown by reactive deposition. $1 \mathrm{ML}$ of iron was deposited in UHV on the substrate kept at room temperature (RT) and then exposed for 5' to $2 \times 10^{-6}$ mbar of $\mathrm{O}_{2}$. After that, with this same partial oxygen pressure, 4 additional ML of iron were evaporated at RT. Finally, the sample was annealed at $675 \mathrm{~K}$ in UHV. Such an annealing temperature was previously used for the growth of $\mathrm{CoO} / \mathrm{Ag}(001)$ [24]. Samples B and C were elaborated by a sequential method consisting in iron deposition, oxidation at RT, and then annealing in oxygen partial pressure. Specifically, sample B was grown following these three steps: First, $5 \mathrm{ML}$ of pure iron were deposited in UHV at RT. After deposition, the layer was dosed with $\sim 10^{-6} \mathrm{mbar}_{2}$ for $\sim 10^{\prime}$ at $\mathrm{RT}$, and finally annealed in $\sim 2 \times 10^{-7}$ mbar $\mathrm{O}_{2}$ at $650 \mathrm{~K}$ for $\sim 30$ '. This is expected to prevent hematite and maghemite growth, these phases having never been reported to our knowledge for annealing in such a low partial oxygen pressure. Sample $C$ was prepared by essentially the same procedure, but its growth was followed step-by-step by in situ SXRD measurements and improved accordingly. Similarly to sample B, about 9 MLs $(1.3 \mathrm{~nm})$ of pure iron were deposited at RT. Then the sample was dosed

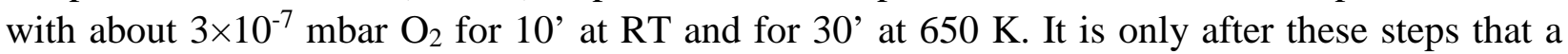
reactive deposition was performed in order to increase the film thickness: Four additional Fe MLs were then evaporated at $650 \mathrm{~K}$ and $3 \times 10^{-7}$ mbar $\mathrm{O}_{2}$ pressure. A final annealing at $770 \mathrm{~K}$ in $\mathrm{O}_{2}$ for 30' was performed before stopping the dosing and switching off simultaneously the heater. This last annealing increases the oxide in-plane domains size as monitored in real time by the width of the characteristic diffraction peaks. It was prolonged until no more structural changes were observed. At the very end of the annealing still no traces of Ag segregation were observed by Auger. The increase in thickness aimed to reduce the weight of the magnetite surface reconstruction contribution on the measured SXRD peak intensities.

\section{Results and Discussion}

\subsection{Growth}


Depending onto the growth conditions, two different crystallographic orientations of the iron oxide epitaxial films are observed without any coexistence. They are well recognized by their LEED pattern, shown in Figure 1. The images are taken at electron energy E=60 eV (a, b) and $70 \mathrm{eV}$ (c). LEED pattern of clean $\mathrm{Ag}(001)$ is presented in Fig. 1a as a reference. The large square (black online) underlines its reciprocal space surface unit cell. Fig 1b shows the LEED recorded for sample A (reactive deposition method) after the final annealing. This pattern results from the superposition of two hexagonal surface meshes rotated by $90^{\circ}$ (diamonds-shaped unit cells, red online) and can be interpreted as arising from two domains of (111)-oriented magnetite. The lattice constant $a_{\mathrm{Fe} 3 \mathrm{O} 4 \text { hex }}$ of its hexagonal surface unit cell is $a_{\mathrm{Fe} 3 \mathrm{O} 4} / \sqrt{ } 2=593.7 \mathrm{pm}$. The epitaxial growth observed here aligns the (100) direction of the $\mathrm{Ag}(001)$ surface mesh (interlayer distance of 288.9 $\mathrm{pm})$ with the $(110)_{\text {hex }}$ direction of the magnetite hexagonal surface mesh (interlayer distance $=$ $\left.a_{\text {Fe304_hex }} \times \cos \left(60^{\circ}\right) / \sqrt{ } 3=296.9 \mathrm{pm}\right)$. The misfit in the rows’ distance is $2.7 \%$ only along this direction. However at $90^{\circ}$ the misfit between $\overrightarrow{a_{s}^{2}}$ and the magnetite $\{200\}_{\text {hex }}$ interlayer spacing is $12 \%$, in agreement with the observed LEED spots separation within the experimental accuracy. Note that after oxygen exposure at RT of the first deposited Fe monolayer we observe only diffuse scattering in addition to weak (1x1) Ag spots. Merte and coworkers studied the RT iron reactive deposition on $\operatorname{Ag}(001)$ in the submonolayer thickness range and showed that after annealing at 675 $\mathrm{K}$ a $\mathrm{FeO}(111)$ epitaxial layer is formed [31]. We conclude that oxidation of Fe/Ag(001) films in the thickness range up to $1 \mathrm{ML}$ favors the (111) orientation.

Unlike sample A, the LEED pattern of sample B (Fig.1c) is consistent with the pattern of a (001)oriented $\mathrm{Fe}_{3} \mathrm{O}_{4}$ film. The magnetite reciprocal space surface unit cell is drawn in the figure with a small square with basis vectors parallel to the Ag ones (blue online). The additional spots come from a $(\sqrt{ } 2 \times \sqrt{ } 2) \mathrm{R} 45^{\circ}$ reconstruction (rotated square mesh, green online). This superstructure is commonly observed on the bulk magnetite surface [32]. Its direct space unit cell coincides with the conventional magnetite one. One should note that (001) maghemite would result in a similar LEED pattern but without the superstructure spots, since it doesn't present a surface reconstruction [33]. This is a first experimental evidence that the grown oxide film is really magnetite. 

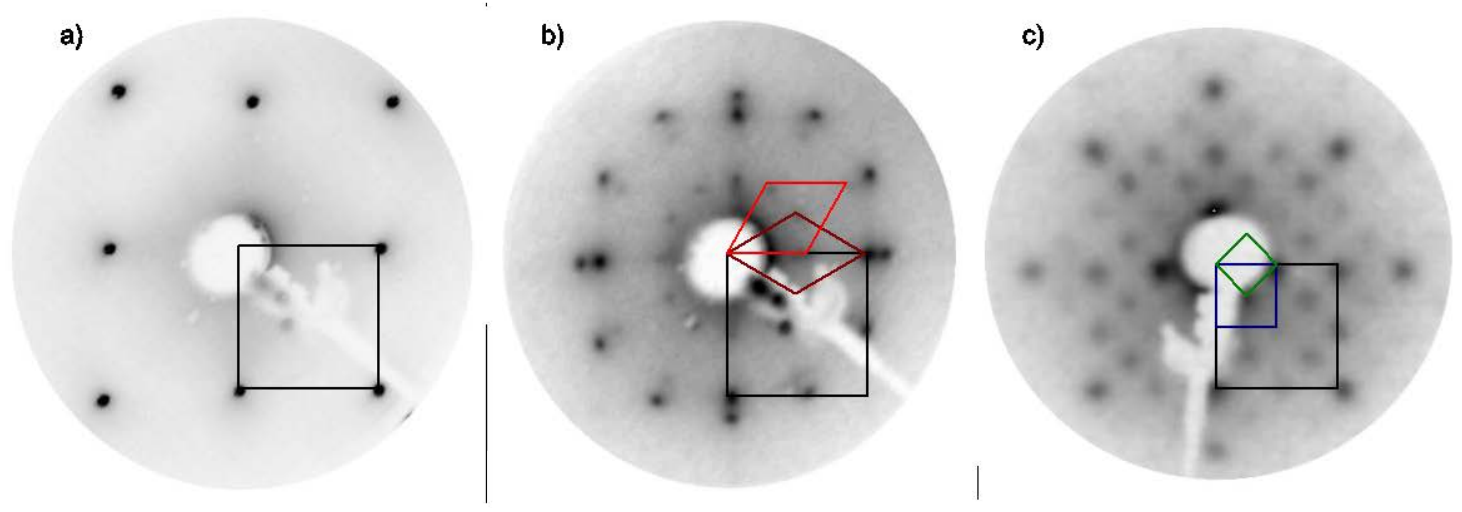

Fig. 1. LEED pattern of: clean $\mathrm{Ag}(001)$ (a); sample A, grown by reactive deposition of Fe in $\mathrm{O}_{2}$ at $\mathrm{RT}$ followed by annealing (b); and sample B, obtained by oxidation of an ultrathin epitaxial Fe layer followed by annealing (c). The images are recorded at $\mathrm{E}=60 \mathrm{eV}(\mathrm{a}, \mathrm{b})$ and $\mathrm{E}=70 \mathrm{eV}(\mathrm{c})$. The large square, the diamond shapes (red online), the medium size square (blue) and the small rotated square (green) represent the $\mathrm{Ag}(001), \mathrm{Fe}_{3} \mathrm{O}_{4}(111), \mathrm{Fe}_{3} \mathrm{O}_{4}(001)$, and $\mathrm{Fe}_{3} \mathrm{O}_{4}(001)$ $(\sqrt{2} \times \sqrt{2}) \mathrm{R} 45^{\circ}$ reciprocal space unit cells, respectively

The film quality (domains size and interface sharpness) has been improved and the preparation conditions optimized by following the growth in real time with in situ SXRD. Figure 2a shows the intensity of $\mathrm{Ag}(11 \mathrm{~L})$ crystal truncation rod (CTR, see ref. [34] for its definition) for sample C at different steps of the growth. The x-axis represents the momentum transfer $\mathrm{Q}_{z}$ perpendicular to the surface $\left(Q_{z}=\left(2 \pi / a_{A g}\right) L\right)$. During Fe evaporation the intensity increases at $\mathrm{Q}_{z}=43.8 \mathrm{~nm}^{-1}$, which is close to the position expected for bulk iron (112) Bragg peak. Fe grows bcc, (001) oriented, and in registry with the substrate. The Fe [100] direction is parallel to $\overrightarrow{a_{s}^{1}}$ in the surface plane. Such in registry epitaxy occurs thanks to the small misfit (0.8\%) between the bulk interatomic distances within the (001) plane, and was already investigated by Heinrich et al. [35 and references therein]. At this stage, a film of about $1.3 \mathrm{~nm}$ was deposited, as evaluated by the period of intensity oscillations at low $\mathrm{Q}_{z}$ (continuous line, red online). Then the film was dosed with $\mathrm{O}_{2}$ at $\mathrm{RT}$, and both the Fe peak and the thickness fringes disappeared (dashed curve, gray online). At the same time the onset of a weak diffraction peak is observed scanning the momentum transfer modulus parallel to the surface (Fig. 2b). It is located at $(\mathrm{H}=0.976, \mathrm{~K}=0.488, \mathrm{~L} \sim 0.49)$ in Ag reciprocal lattice units, about the expected position for (311) magnetite Bragg reflection. These measurements show clearly that the whole film oxidizes and relaxes, forming a seed layer for the growth of a well ordered (001) magnetite layer. 

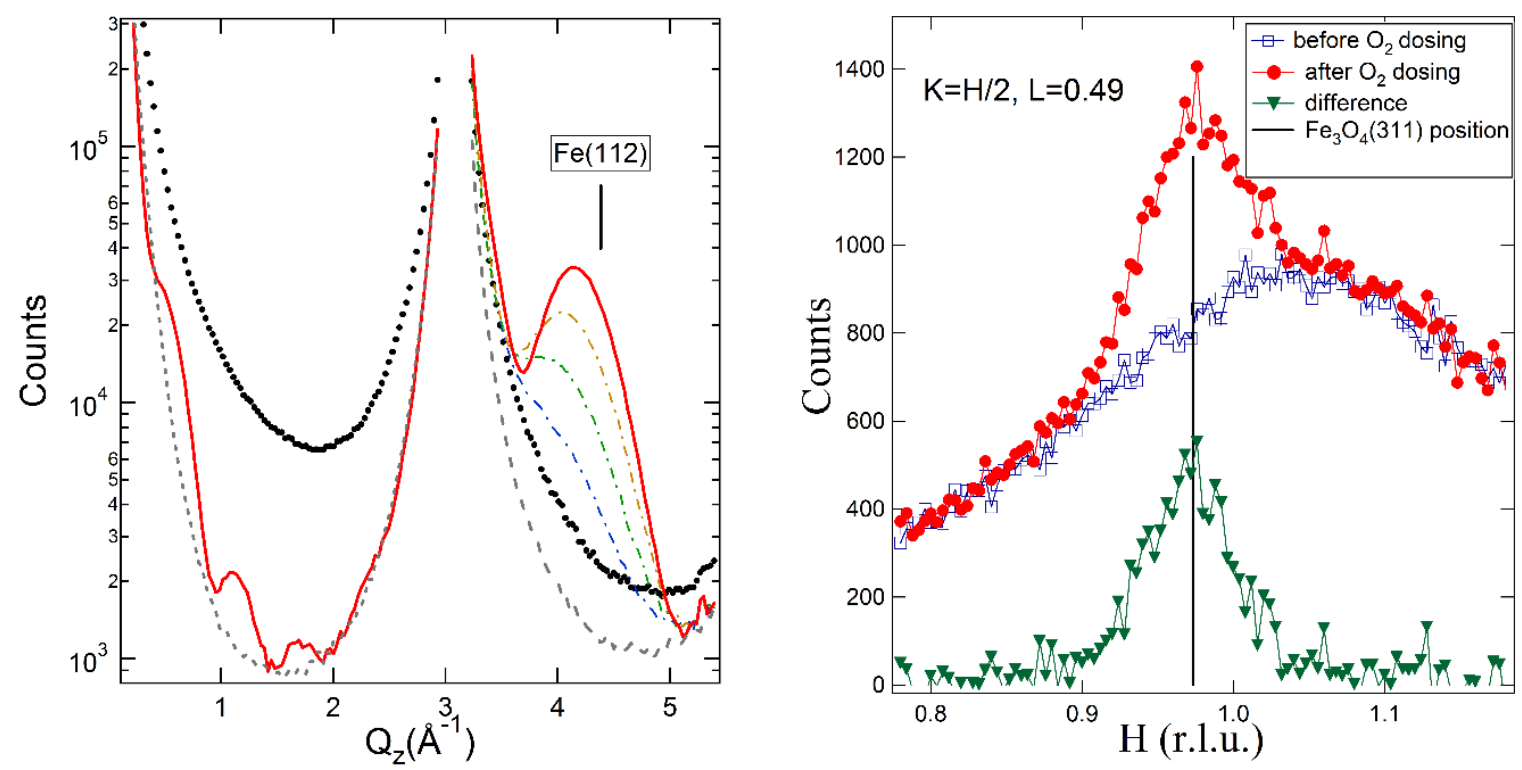

Fig. 2. a) $\mathrm{Ag}(001)$ (11) CTR measured during iron oxide growth. The dotted (black) line refers to clean Ag, the continuous line (red online) to $1.3 \mathrm{~nm}$ of $\mathrm{Fe} / \mathrm{Ag}(001)$, the dot-dashed lines to intermediate thicknesses, and the dashed line is measured after oxidation (gray online), showing the complete disappearing of metallic iron peak. The vertical line indicates the Bragg peak position of bulk-like iron. b) In plane scan through the magnetite (311) peak position before (squares) and after (circles) oxidation of the Fe/Ag(001) film. The difference (triangles) puts in evidence the onset of the magnetite structure peak.

The following annealing in oxygen environment increases the (311) peak intensity by about one order of magnitude reflecting an improvement of the in-plane long-range order. At this stage the oxide film exhibits sharp interfaces, as evidenced by well-defined thickness-fringes observed on the magnetite diffraction rods. Their period gives a film thickness of 2.2+/-0.2 nm. Such a thickness corresponds to the complete oxidation of an iron layer of $1.1+/-0.1 \mathrm{~nm}$ in magnetite. The oxide thickness was increased by further Fe reactive deposition at $650 \mathrm{~K}$. Fig. 3 shows the intensity measured by scanning the momentum transfer perpendicular to the surface in stationary geometry ( $L$-scan) [36] along two magnetite rods, after the final annealing at $770 \mathrm{~K}$ in $\mathrm{O}_{2}$. These $L$-scans cross several magnetite peaks and exhibit Kiessig fringes over the entire measured region, proving the sharpness of both surface and interface. The last annealing increases the characteristic in-plane domains size of the oxide layer to about $25 \mathrm{~nm}$, as found scanning the momentum transfer modulus parallel to the surface. This is large enough for collecting reliable integrated intensities of the magnetite rods. The oxide is very stable and no silver segregation is observed even at such a high temperature, as proved by the absence of its characteristic and strong Auger signal (not shown here). The final thickness is estimated to about $3.4 \mathrm{~nm}$, fitting nicely with an additional deposition of 4 Fe MLs.

The orientation switch observed here in-between the two preparation methods exhibits close analogies with the $\mathrm{CoO} / \operatorname{Ir}(001)$ system [26]. A similar process takes place for magnetite on $\mathrm{Pt}(001)$ [37]. Reactive deposition of iron on $\mathrm{Pt}(001)$ results in highly defective $\mathrm{Fe}_{3} \mathrm{O}_{4}(111)$ films, while reactive deposition on an epitaxial $\mathrm{Fe} / \mathrm{Pt}(001)$ film leads to the formation of $\mathrm{Fe}_{3} \mathrm{O}_{4}(001)$, but only 
if a complete oxidation of the buffer layer is avoided. This should give rise to an ill-defined interface.

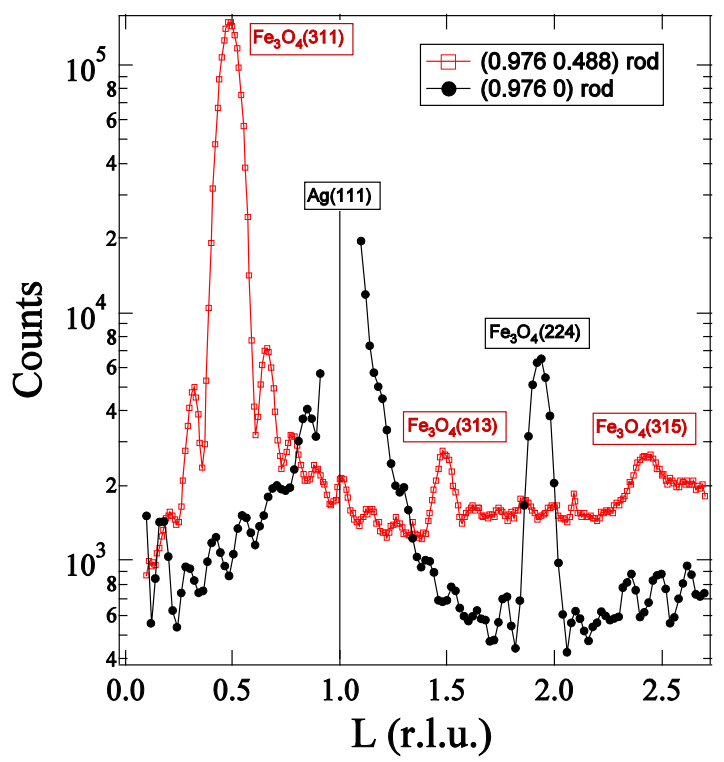

Fig. 3. (0.976 0.488) and (0.976 0) rods. Several peaks of the (001) magnetite film are crossed. Kiessig fringes prove the nice interfaces sharpness.

\subsection{Morphology and Structure}

The two preparation methods described above result in quite different surface morphologies. Fig. 4 shows two 50x50 $\mathrm{nm}^{2}$ STM images of sample A at two different growth stages. In a) the morphology after RT oxidation of the first Fe ML is represented. One can observe that the oxide surface is quite rough, with a root mean square $(\mathrm{rms})$ roughness assessed at $0.29 \mathrm{~nm}$. At the end of the elaboration, after the final annealing at $675 \mathrm{~K}$, the surface still exhibits a rms roughening of $0.26 \mathrm{~nm}$ (Fig 4b). However, this last step increases the lateral correlation length resulting in the appearance of the LEED pattern shown in Fig. 1b. 

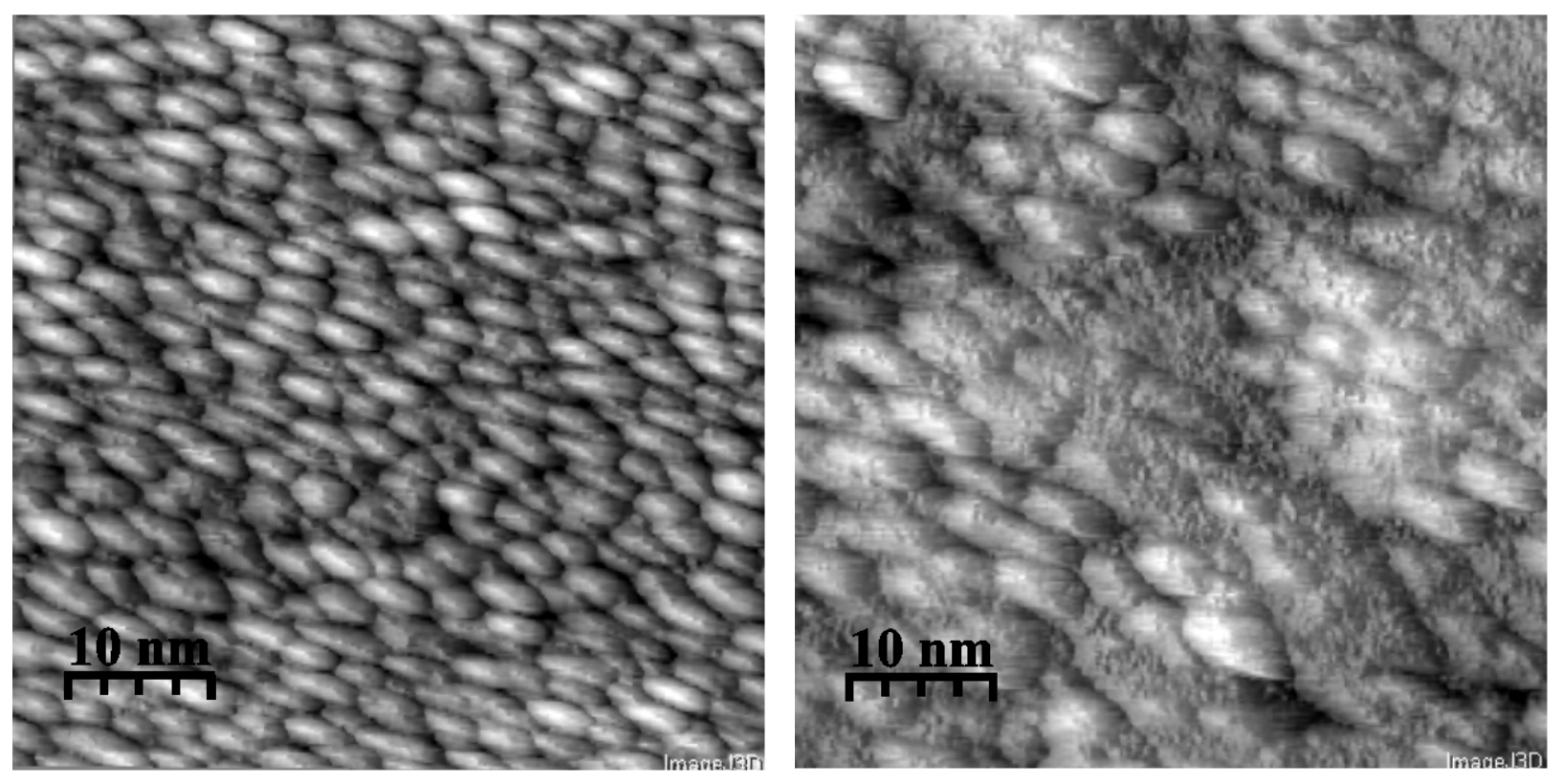

Fig. 4. $50 \times 50 \mathrm{~nm}^{2} \mathrm{STM}$ of : a) $1 \mathrm{ML}$ Fe deposited on $\mathrm{Ag}(001)$ at $\mathrm{RT}$ and exposed to $2 \times 10^{-6} \mathrm{mbar}$ of $\mathrm{O}_{2}\left(\mathrm{~V}_{\mathrm{s}}=2.1 \mathrm{~V}\right.$, $\left.\mathrm{I}_{\mathrm{b}}=0.1 \mathrm{nA}\right)$; b) after further reactive deposition of $4 \mathrm{Fe} \mathrm{ML}$ at RT, followed by annealing at $675 \mathrm{~K}\left(\mathrm{~V}_{\mathrm{s}}=1.2 \mathrm{~V}, \mathrm{I}_{\mathrm{b}}=0.3\right.$ $n A)$.

On the other hand the surface of sample B is quite flat. Its STM image (Fig. 5a) shows terraces wider than $10 \mathrm{~nm}$. In a higher-resolution image (Fig. 5b) atomic rows are observed. They exhibit a wave-like modulation, which is a characteristic of the $(\sqrt{2} \times \sqrt{2}) \mathrm{R} 45^{\circ}$ magnetite surface reconstruction. A first structural model was proposed by Stanka et al. for thick (001) oriented films grown by oxygen-plasma-assisted MBE [32]. The surface termination they obtained is a mixed (001) layer containing oxygen and iron ions in octahedral sites (called B termination). Within this layer, iron atoms are arranged in rows oriented along the $<110>$ direction and separated by 593.7 $\mathrm{pm}$. These are the rows imaged in Fig. 5b. In this model the reconstruction originates from $\mathrm{O}$ vacancies. Later on, based on density-functional theory calculations supported by SXRD measurements, the surface reconstruction was proposed to be the result of a Jahn-Teller effect [38]. More recently a combined LEED, STM and theoretical investigation concluded to a surface structure with an ordered array of subsurface iron vacancies and interstitials, which are at the origin of the wave-like modulation of iron rows [39]. In the following we will consider this model for calculating the surface contribution to the SXRD signal.

In Fig. 5b a domain wall separating two regions of the same terrace with different rows orientation is also observed. It is due to an antiphase domain boundary in the film. Indeed Fe octahedral rows are oriented at $90^{\circ}$ in next magnetite B planes, therefore the row orientation imaged by STM should change at steps only within a single domain. 


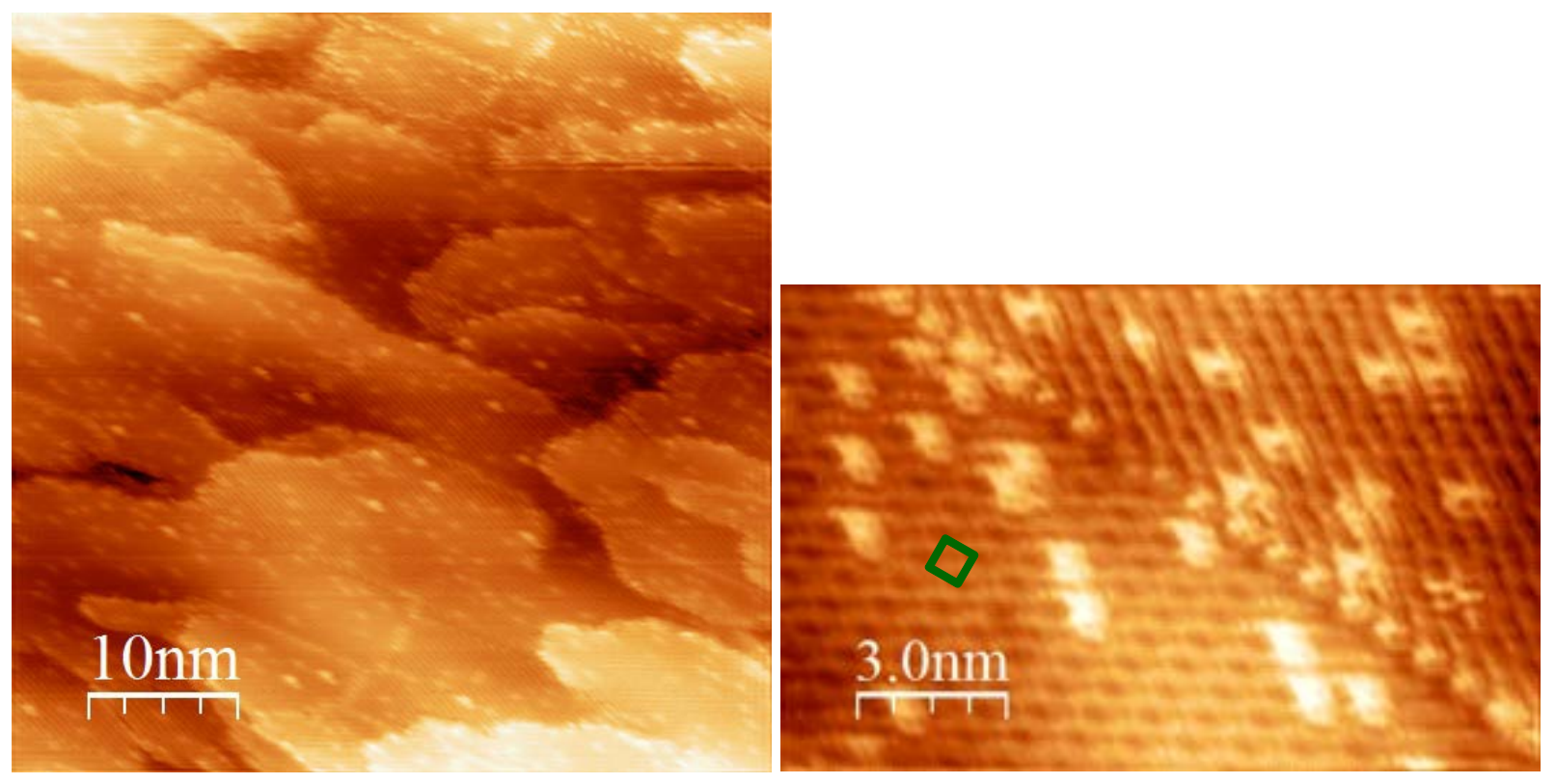

Fig. 5. a) $50 \times 50 \mathrm{~nm}^{2} \mathrm{STM}$ of sample B, obtained by oxidation of an ultrathin Fe layer in epitaxy on $\mathrm{Ag}(001)$ at RT first, and then at $650 \mathrm{~K}\left(\mathrm{~V}_{\mathrm{s}}=1 \mathrm{~V}, \mathrm{I}_{\mathrm{b}}=0.1 \mathrm{nA}\right)$; b) High resolution $\left(15 \times 9 \mathrm{~nm}^{2}\right)$ image of the same sample $\left(\mathrm{V}_{\mathrm{s}}=0.5 \mathrm{~V}\right.$, $\mathrm{I}_{\mathrm{b}}=0.5 \mathrm{nA}$ ). The square (green online) shows the surface reconstruction unit cell (see ref. [32]). Note that the image is not drift corrected, atomic rows should be oriented at $90^{\circ}$ at the domain boundary.

The actual structure of films grown with the three steps method was solved by SXRD. A set of 27 Bragg reflections observed at the expected positions for (111), (311), (511), (202), (422), (113), (224), (115) and equivalents magnetite peaks (according to P4m symmetry), was carefully measured on sample $\mathrm{C}$. Their reciprocal space coordinates were accurately obtained by fitting the spatial intensity distribution along both the parallel and perpendicular directions of the momentum transfer. This set of reflections was used to refine by least square procedures the iron oxide lattice constants and the associated error bars. This procedure permits the observation of a slight tetragonal distortion in the film due to the substrate constraint: $a_{\text {film }}=836.2 \pm 0.3 \mathrm{pm}, c_{\text {film }}=842 \pm 1 \mathrm{pm}$, $c_{\text {film }} / a_{\text {film }}=1.007 \pm 0.002$. The average lattice constants as well as the cell volume are very close to the magnetite values and are slightly larger than the maghemite ones, as reported in Table I.

\begin{tabular}{cccc}
\hline \hline & Film C & Magnetite & Maghemite \\
\hline$a(\mathrm{pm})$ & $836.2 \pm 0.3$ & 839.6 & 833 \\
$c(\mathrm{pm})$ & $842 \pm 1$ & 839.6 & 833 \\
$\mathrm{~V}\left(\mathrm{~nm}^{3}\right)$ & $0.589 \pm 0.001$ & 0.592 & 0.578 \\
$\left(\mathrm{~V}_{\text {film }}-\mathrm{V}_{\text {bulk }}\right) / \mathrm{V}_{\text {bulk }}$ & - & $-0.5 \pm 0.2 \%$ & $1.9 \pm 0.2 \%$ \\
\hline \hline
\end{tabular}

Table I. Film C, magnetite [40] and maghemite [29] lattice constants and unit cell volume.

The experimental intensities of 36 reflections were measured and compared: (i) between themselves to check their deviation from a cubic symmetry, and (ii) with those intensities calculated for the magnetite and maghemite bulk phases. They were collected by rocking the crystal 
around its surface normal and integrated after background subtraction. The corresponding structure factor (SF) values were obtained after correction by the experimental/geometrical factors [36]. Their number reduces to 8 non-equivalent SF after averaging with P4m group symmetry with an agreement factor of $1.3 \%$. This certifies the reliability of the data set. These SF values are reported in Table II. The values for (422) and (224) reflections differ by 10 times their error bar, confirming the deviation from the cubic structure.

In Table II the magnetite and maghemite SF bulk values are reported for comparison. The main difference between film and bulk structures is expected to be related to the presence of the surface reconstruction and to the structural disorder in the nanofilm.

\begin{tabular}{|c|c|c|c|c|}
\hline $\begin{array}{l}\text { Reflection } \\
\text { index }\end{array}$ & Film C & Magnetite & Maghemite & $\begin{array}{l}(\sqrt{2} \times \sqrt{2}) \mathrm{R} 45^{\circ} \\
\text { Magnetite film }\end{array}$ \\
\hline (111) & $25.3 \pm 0.3$ & 24.25 & 9.81 & 27.4 \\
\hline (113) & $100 \pm 1$ & 100 & 100 & 100 \\
\hline (115) & $74 \pm 1$ & 85.13 & 78.35 & 77.5 \\
\hline (202) & $60 \pm 1$ & 62.8 & 68.76 & 60.0 \\
\hline (224) & $49.6 \pm 0.6$ & 49.09 & 52.99 & 47.3 \\
\hline (311) & $98 \pm 1$ & 100 & 100 & 100 \\
\hline (422) & $41.4 \pm 0.5$ & 49.09 & 52.99 & 44.4 \\
\hline (511) & $76 \pm 1$ & 85.13 & 78.35 & 79.5 \\
\hline$\chi^{2}$ & & 35 & 603 & 10.5 \\
\hline
\end{tabular}

Table II. Structure factors measured on sample C compared with magnetite [40] and maghemite [29] bulk ones, calculated from the American Mineralogist crystal structure database [41] applying the standard geometrical corrections. In the last column the comparison with a 4 unit cell thick magnetite film, terminated by the surface reconstruction of ref. [39] is also reported.

The 4 experimental values with lower momentum transfer match qualitatively with both structures, except for the (111) reflection which is incompatible with the maghemite one and is in quite good agreement with the magnetite SF. A quantitative analysis of the ensemble of the reflections results in a normalized $\chi^{2}$ of 35 and 603 for magnetite and maghemite, respectively. The agreement improves considering a 4 unit cells thick magnetite film $(3.37 \mathrm{~nm})$ terminated by the surface reconstruction proposed by Bliem and coworkers. (ref. [39], supplementary materials). The calculation for such a magnetite film is also reported in table II. The presence of the reconstruction breaks the cubic symmetry of the diffraction pattern. Atomic Debye parameters values $B_{i}$ four time larger than in bulk where used, reducing the intensity of large momentum transfer reflections. Such an increase is due to structural disorder and it is an expected feature for an ultrathin film. The discrepancy between measurements and best fit is still statistically significant, but the achievement of a better agreement would require a layer-by-layer fit. A full refinement of the atomic structure cannot be realized due to the large number of atoms contained in the unit cell with respect to the number of measured SF. 
Calculations of magnetite diffraction rods within the Bleim model also reconcile the roughness observed by STM with that one deduced by SXRD. A rms roughness of $0.18 \mathrm{~nm}$ is found from STM measurements reported in Fig. 5a. Calculations performed for ideally truncated magnetite show that a roughness of about $0.3-0.4 \mathrm{~nm}$ is required to explain the Kiessig fringes dumping shown in Fig. 3 (sample C). However the actual shape of diffraction rods is function of the surface structure, in particular when the presence of a reconstruction modifies the electronic density. We actually find that the decrease in the 3rd layer electronic density for the subsurface cation vacancy model gives a substantial contribution to Kiessig fringes amplitude dumping. X-rays are sensitive to a convolution of surface and interface roughness. We can therefore conclude that the magnetitesilver interface is sharp on the atomic layer spacing length scale.

Summarizing, our analysis unambiguously shows that the structure of sample C is very close to a (001) magnetite film. The agreement improves considering the presence of the characteristic $(\sqrt{2} \times \sqrt{2}) \mathrm{R} 45^{\circ}$ magnetite surface reconstruction. A biphasic system can be ruled out, since the presence of a significant maghemite fraction in the film would decrease the agreement with the experimental SF. On the other hand, the presence of a defective iron monoxide phase at the interface would result in a large roughness, which is at variance with the experimental results. In a previous study on this same system [27] a 4nm thick $\mathrm{Fe}_{2} \mathrm{O}_{3}$ layer was first grown and then annealed at $675 \mathrm{~K}$ in UHV first and in oxygen next. This led to a magnetite film, which lacked of the interfaces sharpness and perhaps the single phase requirement indispensable for obtaining the desired electronic properties. The same is probably true for $\mathrm{Fe}_{3} \mathrm{O}_{4}(001)$ grown on $\mathrm{Fe} / \mathrm{Pt}(001)$ [37].

\section{Conclusions}

We have compared two different procedures for the growth of thin iron oxide films in epitaxy on Ag(001). Different crystallographic orientations are obtained depending on the preparation conditions. Oxidation of a two-dimensional Fe layer at room temperature results in a rearrangement of the atomic positions and in the formation of oxide nanoparticles. Increasing the thickness by reactive deposition at RT a rough iron oxide layer is obtained. After annealing at $675 \mathrm{~K}$, a LEED pattern consistent with a (111)-oriented magnetite film is observed.

High quality (001)-oriented magnetite films were grown on $\mathrm{Ag}(001)$ surface following a three step sequential method. It is crucial to start from a predeposited epitaxial Fe layer approximately $1 \mathrm{~nm}$ thick. A seed layer is formed by complete oxidation of such film in a moderate partial oxygen pressure at room temperature. The last step is the annealing in oxygen which leads to the formation of large domains. Once the seed layer is formed, magnetite thickness can be increased by reactive deposition at well-defined temperature and $\mathrm{O}_{2}$ pressure. The magnetite phase was first identified based on qualitative criteria, i.e. the observation of the characteristic $(\sqrt{2} \times \sqrt{2}) \mathrm{R} 45^{\circ}$ surface reconstruction by LEED, and its signature in the STM images: the wave-like modulation along each iron atomic row. Then the presence of a homogeneous magnetite phase with a slight tetragonal distortion and sharp interfaces was determined by quantitative SXRD analysis. In the employed preparation method the formation of other iron oxide phases as well as intermixing, which always 
occurs by annealing the Fe/Ag metallic interface, are avoided. These are advantages for the incorporation of magnetite layers with the desired properties into spintronic devices. The effect of cubic symmetry breaking needs to be further investigated, epitaxial strains being sometimes deliberately used to tailor the physical properties of heteroepitaxial multilayers.

\section{Acknowledgments}

Beamtime is acknowledged at the French CRG BM32 beamline of the ESRF. We thank all the beamline staff and in particular O. Geaymond and O. Ulrich for they invaluable technical support. X. Torrelles thanks the MICINN (Spain) for financial support through Project MAT2012-38213C02-02.

${ }^{*}$ Corresponding author. E-mail address: Maurizio.De-Santis@neel.cnrs.fr

[1] J. S. Moodera, T. S. Santos, T Nagahama J. Phys.: Condens. Matter 19 (2007) 165202.

[2] U. Lüders, M. Bibes, K. Bouzehouane, E. Jacquet E, J.-P. Contour, S. Fusil, J.-F. Bobo, J. Fontcuberta, A. Barthélémy, A. Fert, Appl. Phys. Lett. 88 (2006) 082505.

[3] C. D. Park, D. Magana, A. E. Stiegman, Chem. Mater. 19 (2007) 677.

[4] M. Gich, J. Gazquez, A. Roig, A. Crespi, J. Fontcuberta, J. C. Idrobo, S. J. Pennycook, M. Varela,V. Skumryev, Appl. Phys. Lett. 96 (2010) 112508.

[5]: M. Gich, I. Fina, A. Morelli, F. Sánchez, M. Alexe, J Gàzquez, J. Fontcuberta, A. Roig, Adv. Mater. 26 (2014) 4645.

[6] G. Ketteler, W. Weiss, W. Ranke, R. Schlögl, Phys. Chem. Chem. Phys. 3 (2001) 1114.

[7] F. Walz, J. Phys.: Condens. Matter 14 (2002) R285.

[8] M. Fonin, Y. S. Dedkov, R. Pentcheva, U. Rüdiger, G. Güntherodt, J. Phys.: Condens. Matter 19 (2007) 315217.

[9] M. Friák, A. Schindlmayr, M. Scheffler, New J. of Physics 9 (2007) 5.

[10] S. Yuasa, D. D. Djayaprawira, J. Phys. D: Appl. Phys. 40 (2007) R337.

[11] X. W. Li, A. Gupta, G. Xiao, W. Qian, V. P. Dravid, Appl. Phys. Lett 73 (1998) 3282.

[12] P. Seneor, A. Fert, J.-L. Maurice, F. Montaigne, F. Petroff, A. Vaurès, Appl. Phys. Lett 74 (1999) 4017. 
[13] P.J. van der Zaag, P.J.H. Bloemen, J.M. Gaines, R.M. Wolf, P.A.A. van der Heijden, R.J.M. van de Veerdonk, W.J.M. de Jonge, J. Magn. Magn. Mater. 211 (2000) 301.

[14] Kap Soo Yoon, Ja Hyun Koo, Young Ho Do, Ki Woong Kim, Chae Ok Kim, Jin Pyo Hong, J. Magn. Magn. Mater. 285 (2005) 125.

[15] A.M. Bataille, R. Mattana, P. Seneor, A. Tagliaferri, S. Gota, K. Bouzehouane, C. Deranlot, M.-J. Guittet, J.-B. Moussy, C. de Nadaï, N.B. Brookes, F. Petroff, M. Gautier-Soyer, J. Magn. Magn. Mater. 316 (2007) e963.

[16] Ken-ichi Aoshima, Shan X. Wang, J. Appl. Phys. 93 (2003) 7954.

[17] M. Opel, S. Geprägs, E.P. Menzel, A. Nielsen, D. Reisinger, K.-W. Nielsen, A. Brandlmaier, F.D. Czeschka, M. Althammer, M. Weiler, S.T B. Goennenwein, J. Simon, M. Svete, W. Yu, S.M. Hühne, W. Mader, R Gross, Phys. Status Solidi A 208 (2011) 232.

[18] T. Hibma, F. C. Voogt, L. Niesen, P. A. A. van der Heijden, W. J. M. de Jonge, J. J. T. M. Donkers, P. J. van der Zaag, J. Appl. Phys. 85 (1999) 5291.

[19] J. D. Wei, I. Knittel, U. Hartmann, Y. Zhou, S. Murphy, I. V. Shvets, F. T. Parker, Appl. Phys. Lett. 89 (2006) 122517.

[20] H. Li, Y. Wu, Z. Guo, S. Wang, K. L. Teo, T. Veres, Appl. Phys. Lett. 86 (2005) 252507.

[21] J.-B. Moussy, J. Phys. D: Appl. Phys. 46 (2013) 143001.

[22] M. Luysberg, R. G. S. Sofin, S. K. Arora, I. V. Shvets, Phys. Rev. B 80 (2009) 024111.

[23] T. Kasama, R.E. Dunin-Borkowski, W. Eerenstein, Phys. Rev. B 73 (2006) 104432.

[24] P. Torelli, E.A. Soares, G. Renaud, S. Valeri, X.X. Guo, P. Luches, Surf. Sci. 601 (2007) 2651.

[25] W. Meyer, D. Hock, K. Biedermann, M. Gubo, S. Müller, L. Hammer, K. Heinz, Phys. Rev. Lett. 101 (2008) 016103.

[26] M. Gubo, C. Ebensperger, W. Meyer, L. Hammer, K. Heinz, F. Mittendorfer, J. Redinger, Phys. Rev. Lett. 108 (2012) 066101.

[27] D. Bruns, S. R. Lindemann, K. Kuepper, T. Schemme, J. Wollschläger, Appl. Phys. Lett. 103 (2013) 052401.

[28] M. Canepa, M. Salvietti, A. Campora and L. Mattera, Journal of Electron Spectroscopy and Related Phenomena 76 (1995) 471. 
[29] C. Pecharromàn, T. Gonzàlez-Carregño, J. E. Iglesias, Phys Chem Minerals 22 (1995) 21.

[30] R. Baudoing-Savois, M. De Santis, M.C. Saint-Lager, P. Dolle, O. Geaymond, P. Taunier, P. Jeantet, J.P. Roux, G. Renaud, A. Barbier, O. Robach, O. Ulrich, A. Mougin, G. Bérard, Nucl. Instr. and Meth. in Phys. Res. B 149 (1999) 213.

[31] Lindsay R. Merte, Mikhail Shipilin, Sara Ataran, Sara Blomberg, Chu Zhang, Anders Mikkelsen, Johan Gustafson, Edvin Lundgren, J. Phys. Chem. C 119 (2015) 2572.

[32] B. Stanka, W. Hebenstreit, U. Diebold, S.A. Chambers, Surf. Sci. 448 (2000) 49.

[33] S.A. Chambers, S.A. Joyce, Surf. Sci. 420 (1999) 111.

[34] I.K. Robinson, Phys. Rev. B 33 (1986) 3830.

[35] B. Heinrich, Z. Celinski, J.F. Cochran, A.S. Arnot, K. Myrtle, S.T. Purcell, Phys. Rev B. 47 (1993) 5077.

[36] E. Vlieg, J. Appl. Cryst. 30 (1997) 532.

[37] Earl M. Davis, Ke Zhang, Yi Cui, Helmut Kuhlenbeck, Shamil Shaikhutdinov, HansJoachim Freund, Surface Science 636 (2015) 42.

[38] R. Pentcheva, F. Wendler, H. L. Meyerheim, W. Moritz, N. Jedrecy, M. Scheffler, Phys. Rev. Lett. 94, (2005) 126101.

[39] R. Bliem, E. McDermott, P. Ferstl, M. Setvin, O. Gamba, J. Pavelec, M. A. Schneider, M. Schmid, U. Diebold, P. Blaha, L. Hammer, G. S. Parkinson, Science 346 (2014) 1215.

[40] B.A. Wechsler, D.H. Lindsley, C.T. Prewitt, American Mineralogist 69 (1984) 754.

[41] R.T. Downs, M. Hall-Wallace, the American Mineralogist Crystal Structure Database. American Mineralogist 88 (2003) 247. 